\title{
USEP: Ultra Stable Threshold Sensitive Election Protocol for Mobile WSN
}

\author{
Surendra Verma \\ Research Scholar \\ Electronics \& Communication Engineering \\ Department \\ Mewar University, Chittorgargh India
}

\author{
K.C.Mahajan, Ph.D \\ Professor \& Principal \\ MIT Bhopal \\ Madhya Pradesh, India
}

\begin{abstract}
Wireless sensor networks (WSN's) are going to replace old mode of information collection in various area like medical, industry, communication. In this paper authors propose a new Ultra stable Threshold sensitive election protocol (USEP), which is a reactive routing protocol using five level heterogeneity. Reactive networks are fast in response then proactive network. Here protocol has been tested for variation in temperature sensing application.
\end{abstract}

\section{General Terms}

Hope, route, node, sensor, energy, base station

\section{Keywords}

WSN, SEP, LEACH, TEEN, Cluster.

\section{INTRODUCTION}

The wireless sensor network is latest trend for social development and data collection technology. In recent years, advances in miniaturization; low-power circuit design; simple, low power, yet reasonably efficient wireless communication equipment; and improved small-scale energy sup- plies have combined with reduced manufacturing costs to make a new technological vision possible: Wireless sensor networks.

The main challenge for Mobile WSN is efficient and authenticated energy transmission among nodes and between hopes. There are several algorithm available for improvement of routing methods, energy conservation and timely delivery of data packets.

Most prominent routing algorithms can be categorized into three types i.e direct transmission algorithms, hop to hop transmission algorithms and cluster based algorithms.

Second challenge is that persists is to handle heavy information sensed and passed over by every node of a network. (A WSN may consist of hundred thousands of nodes). For that data collection, aggregation and data fusion algorithms work, however there is always a room for betterment.

Authors have done complete analyses in their work about cluster based routing of wireless sensor networks. Later they have presented novel \& improved modifications which are based on the platform of most prominent routing protocol. The results have been compared with other protocols output performance.

\section{BACKGROUND}

Lot of efforts were made by researchers worldwide in WSN arena. The cost effective version of WSN having good computation and low power transmission capability are now easily available. Though lot of scope of improvement is there, but growth rate of wireless sensor network area is conspicuous.

It has been observed widely that power, bandwidth consummation are main constraints for further growth. In direct transmission, a node sense data from its environment and transmits it straight to base center (BS). The direct method of transmission is no doubt, ensures data security, on the other hand it compromises on life time due to excessive power consumption. Hence, nodes far from BS dies early, as they needs more power to propagate their signal.

Estrin et. al [3] worked on an hierarchical clustering mechanism dealing with asymmetric communication for power saving in sensor nodes. Jiang et.al presented a cluster based routing protocol (CBRP) [4]. According to this mechanism, all participating nodes of network are distributed in 2-hop cluster. Though this protocol is not much energy efficient for wireless sensor nodes however, it gives way to hierarchical clustering algorithms. Clustering for energy conservation is proven as efficient mechanism for wireless sensor networks [5].

\section{MOTIVATION}

The main target is to improve existing protocols in terms of throughput and routing target selections. The basic LEACH algorithm with TEEN algorithm's threshold selection is prime focus. According to LEACH rule for every round, new cluster head is elected and hence new cluster formation is required. This causes unnecessary excessive energy consumption. If a cluster head is not able to utilize much of its energy in current round, of there is high probability of replacement in next round by some low energy nodes, in head election process.

The target is to stop unnecessary change in cluster head, after every round. Hence a proficient algorithm for head replacement is required [1]. LEACH algorithm uses constant amount of amplification energy to transmit data regardless of distance between two sides. To save energy, there should be a transmission process that specify required amplification energy for interacting with head or base node. We mean to say that variable amount of amplification energy must be used for process. The global look up table format for energy requirement may not be a good solution as it will enhanced required routing complexities.

Jived \$ Mahmood [1] have proposed two solutions: efficient cluster head replacement and dual transmitting power level. They have also demonstrated multi-level division of optional probabilities of nodes, which are divided on the basis of energy, to be elected as a $\mathrm{CH}$ can be calculated by using specific formulas [2]. We propose combination of these two algorithm and further division of node energy parameters. 


\section{PROPOSED ALGORITHM}

In LEACH algorithm, which is proactive routing protocol proposed in [], thousands of nodes dispersed randomly for even distribution of load among nodes. To divide burden among nodes, improved network life clusters are formed.

The channel head $(\mathrm{CH})$ threshold calculation formula may be given as $T_{n}=\left\{\frac{p}{1-p\left[\operatorname{r.mod} \frac{1}{p}\right]}\right\}$

In stable election protocol heterogeneity is introduced based on two levels. A fraction of $\mathrm{m}$ of $\mathrm{n}$ nodes is provided with an additional energy factor $\alpha$, which are called advanced nodes.

So probabilities of normal and advanced nodes are

$$
\begin{aligned}
& P_{n n r m}=\left\{\frac{p_{o p t}}{[1+m \alpha]}\right\} \\
& P_{n a d v}=\left\{\frac{p_{o p t}(1+\alpha)}{[1+m \alpha]}\right\}
\end{aligned}
$$

Where $p_{\text {opt }}$ is optional probability of each node to become $(\mathrm{CH})$. By increasing $p_{a d v}$ or $m$ we can further improve system performance. The $p_{o p t}$ and $m$ should be correlated. To describe complete solution we would like to mention types of nodes. For multilevel heterogeneity, nodes with different energy levels are
1) Normal Nodes
2) Sub Normal Nodes
3) Intermediate Nodes
4) Advance Nodes
5) Super Nodes

Super nodes having energy level greater than all other nodes. Remaining nodes have energy level as per order only. The intermediate nodes can be chosen by using, a fraction of nodes which are intermediate nodes and using the relation that intermediate node energy is $\mu$ times more than that of normal and sub normal nodes.

$$
\begin{gathered}
E_{A D V}=E_{0}(1+\alpha), \text { is advance node energy, } \\
E_{\text {int }}=E_{0}(1+\mu) \text { where } \quad \mu=\alpha / 2
\end{gathered}
$$

So total energy of all these nodes will be $\eta b(1+\alpha)$, $\eta E_{0}(1-m \alpha-b n)$, and $\eta m E_{0}(1+\alpha), \eta E_{0}(1+$ $m \alpha+b n) \eta E_{0}(1+2 m \alpha+b n)$ respectively.

Where $\mathrm{n}$ is the number of nodes, $\mathrm{m}$ is proportion of advanced nodes to total number of nodes $n$ with energy more than rest of nodes and $\mathrm{b}$ is proportion of intermediate nodes.

The optimal probability of nodes, which are on the basis of energy, to be selected as channel head, can be calculated as

$$
\begin{aligned}
& P_{\text {nnrm }}=\left\{\frac{p_{o p t}}{[1+m \alpha+\mathrm{b} \mu]}\right\} \\
& P_{\text {subnrm }}=\left\{\frac{p_{o p t(1+\mu)}}{[1+m \alpha+\mathrm{b} \mu]}\right\} \\
& P_{\text {int }}=\left\{\frac{p_{o p t}(1+2 \mu)}{[1+m \alpha+\mathrm{b} \mu]}\right\}
\end{aligned}
$$

$$
\begin{aligned}
& P_{a d v}=\left\{\frac{p_{o p t}(1+\alpha)}{[1+m \alpha+\mathrm{b} \mu]}\right\} \\
& P_{\text {super }}=\left\{\frac{p_{o p t}(1+2 \alpha)}{[1+m \alpha+\mathrm{b} \mu]}\right\}
\end{aligned}
$$

To ensure that $\mathrm{CH}$ election is done in the same way, as it was assumed, authors have taken another parameter into consideration, which is threshold level. Each node generates randomly a number inclusive of 0 and 1 , if generated value is less than critical value then this node becomes $\mathrm{CH}[1][2]$. For all these categories there are separate formulas for the calculation of threshold depending on their probabilities, which are given below::

$$
\begin{aligned}
& T_{\text {nrm }}=\left\{\frac{p_{\text {nrm }}}{\left[1-\text { Prnm }\left[\text { r.mod } \frac{1}{p_{\text {nrm }}}\right]\right.}\right\} \\
& T_{\text {int }}=\left\{\frac{p_{\text {int }}}{\left[1-\text { Pint }\left[\text { r.mod } \frac{1}{p_{\text {int }}}\right]\right.}\right\} \\
& T_{\text {adv }}=\left\{\frac{p_{\text {adv }}}{\left[1-\text { Padv }\left[\text { r.mod } \frac{1}{p_{a d v}}\right]\right.}\right\} \\
& T_{\text {sup }}=\left\{\frac{p_{\text {sup }}}{\left[1-\text { Psup }\left[\text { r.mod } \frac{1}{p_{\text {sup }}}\right]\right.}\right\} \\
& T_{\text {subn }}=\left\{\frac{p_{\text {subn }}}{\left[1-\text { Psubn }\left[\text { r.mod } \frac{1}{p_{\text {subn }}}\right]\right.}\right\}
\end{aligned}
$$

Average total number of $\mathrm{CHs}$ per round will be:

$$
\begin{array}{r}
n(1-m-b) p_{n r m}+n \cdot b \cdot p_{i n t}+n \cdot m \cdot p_{a d j} \\
+n(1+m+b)=n \cdot p_{o p t}
\end{array}
$$

Although, average number of $\mathrm{CHs}$ is same as that of LEACH or SEP. However, In case of our algorithm there is a good aspect of energy dissipation in right magnitude.

At the start of each round, here takes place the phenomenon of cluster change. In case of USEP, at cluster change time $\mathrm{CH}$ broadcasts the following parameters higher threshold, lower threshold, report time, attributes and energy level of cluster head. All nodes keep on sensing environment continuously .As parameters from attribute set reaches higher threshold, transmitter is turned on and data is transmitted to $\mathrm{CH}$, however this is for the first time when this condition is met. This value will be stored. Then for next time and future nodes will transmit data if and only if sensed values is greater than high threshold value. So by keeping all this in consideration, number of data transmission can be reduced, as transmission will only take place when sensed value reached high threshold value.

Main features are given below:

i) Time critical data reaches the user almost instantaneously 
ii) Nodes keep on sensing continuously but transmission is not done frequently, so energy consumption is much more less than that of proactive networks.

iii) At time of cluster change, values of soft threshold, report time and $\mathrm{A}$ are transmitted afresh and what parameters to be sensed according to the criticality of sensed attribute and application.

iv) The user can change the attributes depending on requirement, as attribute are broadcasted at the cluster change time.

\section{SIMULATION}

The simulation has been done in Matlab R2009a. For verification algorithm was tested on the basis of five point calculation.

1) The period from the start of the network operation and the first node.

2) The period between the first dead node and last dead node.

3) Number of alive nodes per round

4) Number of dead nodes per round

5) Throughput, number of packets sent from cluster heads to base station.

A network consisting of 110 nodes, placed randomly in a region of MxM and a BS located in the center is considered. We performed simulations for different values of $\alpha$ and $m$ while keeping $b$ constant that is 0.4 . For the first case $\alpha=1, m=$ 0.2 , for second case $\alpha=4$ and $m=0.3$. This is done to observe change in network's stability, life and throughput relative to increase in number of advance nodes and their energies. Since $\mathrm{P}_{\mathrm{opt}}=0.1$, is the optimal probability of $\mathrm{CHs}$, by using equations (4), (5) and (6), (7) \& (8) we obtained different probabilities for each type of nodes in accordance with different values of $\alpha$ and $m$. Other parameters used in simulations are given below

$E_{\text {elect }}$, efs $, E_{D A}, \varepsilon m p, E, \mathrm{~K}, \quad$ Popt, $\eta, \alpha, \mathrm{m}$ and their respective values are as:

\begin{tabular}{|c|l|l|}
\hline Parameters & \multicolumn{1}{|c|}{ SET1 } & \multicolumn{1}{|c|}{ SET2 } \\
\hline$E_{\text {elect }}$ & $50 \mathrm{~nJ} / \mathrm{bit}$ & $70 \mathrm{~nJ} / \mathrm{bit}$ \\
\hline$e f s$ & $5 \mathrm{~nJ} / \mathrm{bit} / \mathrm{message}$ & $8 \mathrm{~nJ} / \mathrm{bit} / \mathrm{message}$ \\
\hline$E_{D A}$ & $10 \mathrm{jj} / \mathrm{bit} / \mathrm{msq}$ & $20 \mathrm{pj} / \mathrm{bit} / \mathrm{msq}$. \\
\hline$\varepsilon m p$ & $.0013 \mathrm{j} / \mathrm{bit} / \mathrm{m} \mathrm{sq}$ & $.0018 \mathrm{j} / \mathrm{bit} / \mathrm{m} \mathrm{sq},$. \\
\hline$E$ & $5 \mathrm{j}$ & $10 \mathrm{j}$ \\
\hline $\mathrm{K}$ & 4000, & 6000 \\
\hline Popt & 0.1 & 0.2 \\
\hline$\eta$ & 100 & 100 \\
\hline & &
\end{tabular}

\begin{tabular}{|l|l|l|}
\hline$\alpha$ & 1 & 1 \\
\hline $\mathrm{m}$ & 0.1 & 0.2 \\
\hline
\end{tabular}

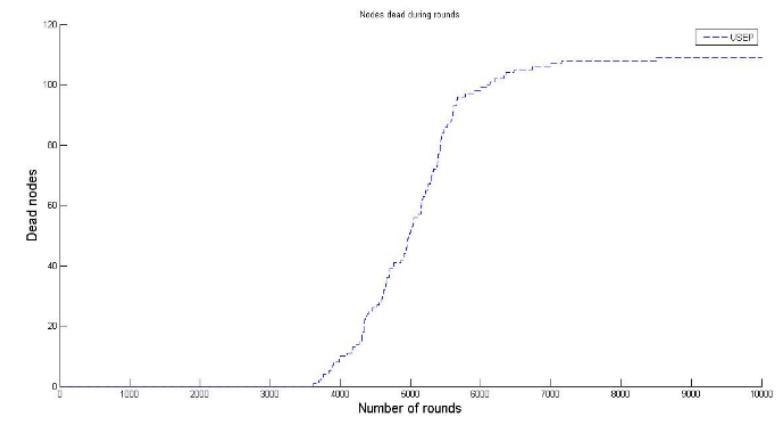

Figure 1. Dead Nodes vs Iterations, SET1

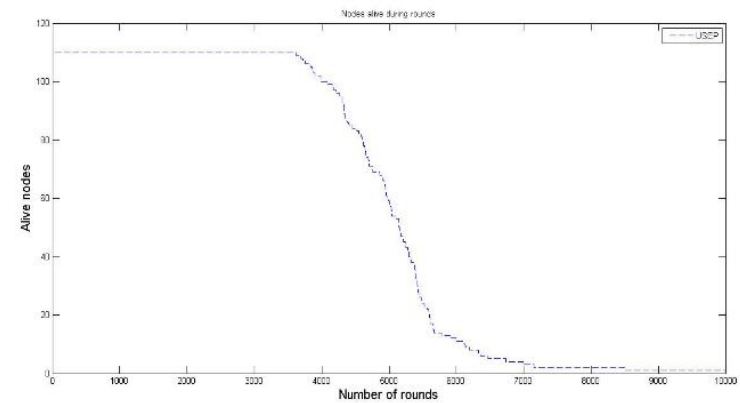

Figure 2: Nodes alive during rounds vs Iterations, SET1

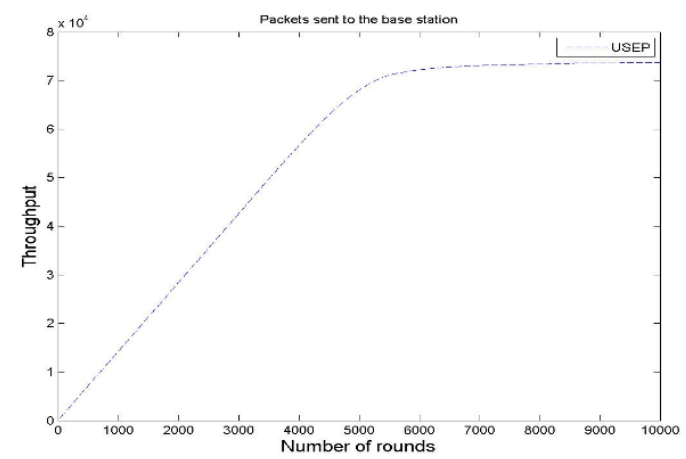

Figure 3: Throughput Vs Iterations, SET1

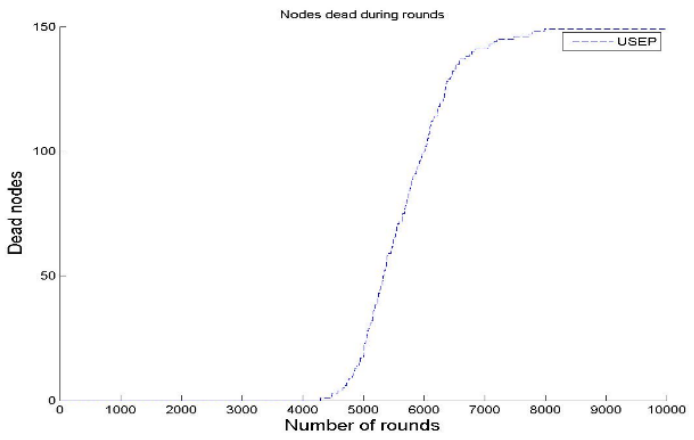

Figure 4: Dead nodes Vs Iterations, SET2 


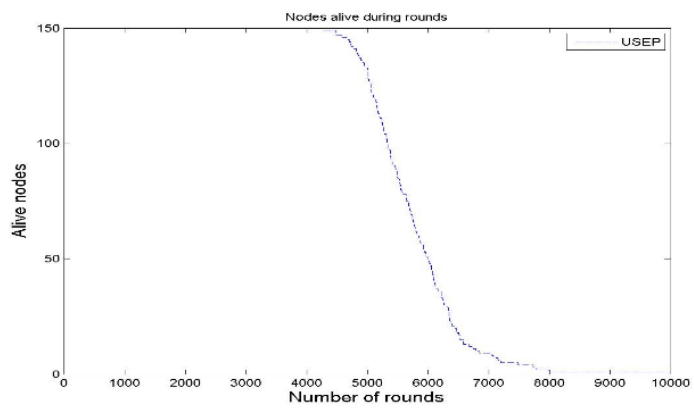

Figure 5 : Nodes alive during round Vs Iterations, SET2

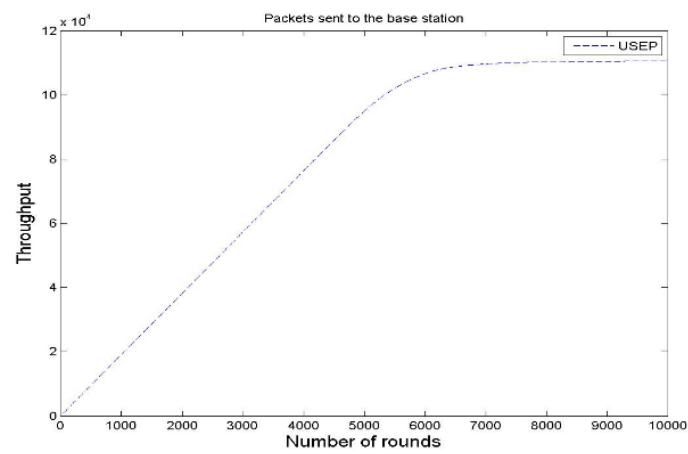

Figure 6 : Throughput Vs Iterations, SET2

By performing simulation in MATLAB, it is observed that: USEP has enhanced period than all previous than all other prototypes. The network life for USEP was increased as compared to others. Increased through put due to five level heterogeneity and decrease in throughput due to sensitivity.

\section{CONCLUSION}

In this paper USEP, reactive protocol is proposed where nodes with five level of energies .Channel heads selection is threshold based, due to five level of heterogeneity and being reactive routing network protocol, it causes increment in stability and network life, which is better than TEEN,SEP,LEACH protocols.

It is novel routing algorithm for WSN's. This can be performed on network simulators and UNIX platform. The higher up gradation in probability calculation and channel selection will be added challenge for upcoming algorithms.

\section{REFERENCES}

[1] Kashaf A., Javaid N, .Khan "TSEP :Threshold-sensitive stable election Protocol for WSNS" in International journal of science and Technology ,17 Dec 2012 ,p.p.41

[2] T. J. Shepard, "A channel access scheme for large dense packet radio networks." in Proceedings of ACM
SIGCOMM, September 1996, p p. 219230.

[3] Heinzelman, Chandrakasan, and Balakrishnan. "EnergyEfficient Communication Protocols for Wireless Micro sensor Networks." In Proceedings of Hawaiian International Conference on Systems Science, January 2000.

[4] Smaragdakis, Matta, Bestavros, "SEP: A Stable El election Protocol for clustered heterogeneous wireless sensor networks." in : Second International Workshop on Sensor and Actor Network Protocols and Applications (SANPA 2004), 2004.

[5] Femi A. Aderohunmu, Jeremiah D. Deng,“ An Enhanced Stabl e Election Protocol (SEP) for Clustered Heterogeneous WSN." Department of Information Science, University of Otago, New Zealand.

[6] Manjeswar, A.; Agrawal, D.P. "TEEN: A protocol for enhanced efficiency in wireless sensor networks." In Proceedings of 1st Interna- tional Work-shop on Parallel and Distributed Computing Issues in Wireless Networks and Mobile Computing, San Francisco, CA, USA, 2001; p. 189.

[7] "An application-specific protocol architecture for wireless microsensor-networks," IEEE Transactions on Wireless Communications, vol. 1, no. 4, pp. 660670, October 2002.

[8] S. Bandyopadhyay and E. J. Coyle, "An energy efficient hie- rarchical clustering algorithm for wireless sensor networks," in Pro ceedings of INFOCOM 2003, April 2003.

[9] [9] S. Bandyopadhyay and E.J. Coyle, "Minimizing communication costs in hierarchically-clustered networks of wireless sensors," Computer Net-works, Vol. 44, Issue 1, pp. 1-16, January 2004.

[10] Li Qing, Qingxin Zhu, Mingwen Wang, "Design of a distrib uted energy-efficient clustering algorithm for heterogeneous w ireless sensor networks," Computer Communications, Volume 29, Issue 12, 4 August 2006, Pages 2230-2237.

[11] W. R. Heinzelman, A. P. Chandrakasan, and H. Balakrishnan "An application-specific protocol architecture for wireless micro sensor net-works," IEEE Transactions on Wireless Communications, vol . 1, no. 4, pp. 660670, October 2002.

[12] C. Siva Ram Murthy and B.S Manoj, "Ad Hoc Wireless Networks Architectures and Protocols." Prentice Hall Communication Engineering and Emerging Technologies Series, 2004. 